\title{
Periodic phenomena on a partially cavitating hydrofoil
}

\author{
Anne Gosset \\ Universidad de A Coruña \\ Ferrol, Spain \\ anne.gosset@udc.es
}

\author{
Marcos Lema \\ Universidad de A Coruña \\ Ferrol, Spain \\ marcos.lema@udc.es
}

\author{
Fernando López Peña \\ Universidad de A Coruña \\ Ferrol, Spain \\ flop@udc.es
}

\section{ABTRACT}

This paper aims at evaluating the sensitivity of the numerical simulations of patch and sheet/cloud cavitation to turbulence modeling in 2D. The periodic phenomena related to cavity shedding and cloud formation on a partially cavitating NACA0015 foil are studied within a wide range of conditions, on relatively coarse meshes. The finite volume CFD package OpenFoam is used. A Homogeneous Equilibrium Approach is employed, in which cavitation modeling is achieved through a transport equation for the vapor volume fraction. The effect of turbulence modeling is analyzed, comparing the results yielded by Implicit Large Eddy Simulation (ILES) and a URANS k- $\omega$ SST model in two versions: the conventional one of Menter, and a modified one in which turbulent viscosity is made sensitive to vapor fraction. The cavity dynamics is well captured with ILES, and its dominant frequencies are well validated with experimental data. As for the RANS models, it is found as expected that the k- $\omega$ SST in its original formulation leads to an unrealistic stabilization of the cavity. With the modified version, the dynamics of the cavity is triggered in the sheet/cloud regime, and the periodic character of cavity shedding/cloud formation is well captured. However, the frequency of the process is a little lower than with ILES, and close to patch cavitation, the modification is not sufficient to trigger a substantial dynamics. This is due to the smaller cavity volumes predicted by the k- $\omega$ SST model, which delays slightly shedding. Through processing of the flow fields, it is demonstrated that the frequency of the travelling and collapsing vapor clouds is related to the frequency of cavity shedding.

\section{INTRODUCTION}

The current state of knowledge in the CFD simulation of cavitation makes it possible to describe accurately the cavitating flow in a number of classical test cases, such as the flow past a NACA0015 hydrofoil [1-2]. But the high-fidelity prediction of the variety of dynamic phenomena observed on a foil (reentrant jet, cavity shedding and cloud formation, upstream desinence, secondary cavities...) involves a CPU cost that is not always compatible with the use of these numerical methods in optimization processes, or larger scale simulations (e.g. full propellers). Because these dynamic phenomena, in particular the collapse of vapor clouds, are directly related to the rate of erosion [3], it is crucial to be able to predict their occurrence, even with low accuracy, in order to estimate the erosive risk.

In single fluid approaches, the turbulence modeling applies to the entire field of vapor-liquid mixture, in such a way that mass transfer models are tightly coupled with turbulence modeling when it comes to simulate highly unsteady cavitating flows [4]. It can be expected that URANS are able in essence to predict large scale motions such as sheet formation and large cavity shedding, but it should be able also to predict essential smaller scale phenomena such as the formation of reentrant jet and cloud breakup which are crucial for the global dynamics of cavitation. The formation, transport, and collapse of vapor clouds is indeed determinant for evaluating the risk of erosion. Bernsten et al. [5] show that the lack of turbulence modeling leads to erroneous predictions of the cavity shedding frequency in the sheet cavitation regime. Coutier-Delgosha et al. [6] show that standard RANS models such as k- $\varepsilon$ RNG and k- $\omega$ associated to a barotropic law for phase change are not able to capture the dynamics of cavitation in a Venturi section. They propose to make the turbulent viscosity sensitive to void fraction in the k- $\varepsilon$ RNG, and to include compressibility effects in the vapor-liquid mixture in the $\mathrm{k}-\omega$ model. These two modifications lead to a better prediction of the reentrant jet, which triggers a periodic cavity shedding of similar frequency in both cases. This modification of the k- $\varepsilon$ RNG model is later validated [7] on a 2D symmetric hydrofoil which served as a benchmark test case in CAV2003. Pouffary et al. [8] apply a similar correction on a standard $\mathrm{k}-\varepsilon$ model and obtain comparable results for the same test case. In contrast, Kunz et al. [9] succeed in capturing the dynamics of cavitation with a 
standard k- $\varepsilon$ model and a barotropic law. Their DES simulations of the same test case give rise to larger cavities and reveal a much richer dynamics, even if the authors underline the heavy CPU requirements. Kim [2] finds also that at low cavitation numbers, the standard k- $\omega$ and k- $\varepsilon$ Realizable models allow retrieving the strong unsteadiness of sheet/cloud cavitation on a NACA0015 profile, even if no periodic pattern is detected. 3D LES and DES computations yield better predictions of the flow periodicity, in good agreement with experiments. However, the computational cost involved in such simulations is considerable if one wants to apply the lessons learned on larger 3D configurations, such as marine propellers. In this paper, we propose to characterize the dynamics of cavitation on a 2D NACA0015 hydrofoil at a modest computational cost, retaining the most crucial phenomena such as the formation and collapse of vapor clouds. An emphasis is given to the periodic mechanisms related to cavity shedding and cloud collapsing, whose characteristics are studied systematically over a wide range of conditions with standard and modified k- $\omega$ SST models, and Implicit Large Eddy Simulation (ILES). The study aims at evaluating the feasibility of relatively "cheap" models in $2 \mathrm{D}$, in order to choose an approach for future 3D computations.

\section{NUMERICAL MODELING AND SIMULATION SETUP}

The CFD simulations are conducted with the open source CFD package OpenFoam. They are based on a cell-centered Finite Volume technique with a PISO algorithm for velocitypressure coupling. Cavitation is modeled through a single fluid, two-phase mixture approach, introducing the liquid volume fraction $\gamma$, which is used to scale the mixture physical properties:

$\rho=\gamma \rho_{v}+(1-\gamma) \rho_{l}$

$\mu=\gamma \mu_{v}+(1-\gamma) \mu_{l}$

Using a VOF approach, a transport equation for $\gamma$ is added to the mass and momentum equations. A non-compressive reconstruction scheme is used for the convection of the volume fraction. Vaporization and condensation appear as source terms in the continuity (3) and $\gamma$ equation (4):

$\nabla \cdot \overrightarrow{\boldsymbol{v}}=\dot{m}\left(\frac{1}{\rho_{l}}-\frac{1}{\rho_{v}}\right)$

$\frac{\partial \gamma}{\partial t}+\nabla \cdot(\gamma \overrightarrow{\boldsymbol{v}})=\dot{m} / \rho_{v}$

where $\dot{m}=\dot{m}_{c}+\dot{m}_{v}$ is the net mass transfer rate. The rates for condensation and vaporization are treated separately, and evaluated with a model based on bubble dynamics derived from Schnerr et al. [10], implemented in OpenFoam in the form:

$\dot{m}_{c}=C_{c} \frac{3 \rho_{v} \rho_{l}}{\rho} \frac{\gamma(1-\gamma)}{R_{b}} \operatorname{pos}\left(p-p_{v}\right) \sqrt{\frac{2}{3} \frac{\left|p-p_{v}\right|}{\rho_{l}}}$

$\dot{m}_{v}=-C_{v} \frac{3 \rho_{v} \rho_{l}}{\rho} \frac{\gamma(1-\gamma)}{R_{b}} \operatorname{pos}\left(p-p_{v}\right) \sqrt{\frac{2}{3} \frac{\left|p-p_{v}\right|}{\rho_{l}}}$

where $p_{v}$ is the vapor pressure of the liquid and $R_{b}$ the bubble radius. A sensitivity study of the results to the coefficients $C_{c}$, $C_{v}$ and $R_{b}$ led us to use the following values: $C_{c}=2, C_{v}=1$, $R_{b}=10^{-4} \mathrm{~m}$, with a number of nuclei per unit volume $N_{b}$ equal to $2 \times 10^{8}$. More details on the solver can be found in $[1,2]$.
The computations are run with two URANS models: a conventional k- $\omega$ SST model, and a k- $\omega$ SST modified to decrease turbulent viscosity in the vapor-liquid regions. The rest of the simulations are conducted with ILES, as in [1]. In the classical filtered equation of LES, the unresolved term in the subgrid stress tensor is not expressed using an explicit model as in LES; numerical dissipation is assumed to simulate the physical dissipation generated by spatial subgrid scales.

The simulations are conducted on a 2D NACA0015 hydrofoil, known for being a very classical test case. A uniform velocity $U=8 \mathrm{~m} / \mathrm{s}$ is imposed at the inlet of the flow domain, while the pressure at the outlet $p_{\text {out }}$ is fixed at 0 . Symmetry conditions are used at the bottom and top boundaries. The flow Reynolds number based on the chord length $c$ is $6.5 \times 10^{5}$, and the cavitation number $\sigma=\left(p_{\text {out }}-p_{v}\right) / 0.5 \rho_{l} U^{2}$ and angle of attack $\alpha$ are varied in order to simulate a wide range of cavitation regimes. The baseline mesh for RANS simulations contains 23000 cells, mesh sensitivity was checked with a finer mesh of 40000 cells. In most computations, the mesh resolution at the wall is adjusted to get a $y^{+}$between 40 and 80 , and a continuous adaptive wall function is used, in a similar fashion than with the Spalart-Allmaras model. The finer mesh allowed reaching $y^{+}$between 6 and 10 . This approach is necessary since the k- $\omega$ SST in OpenFoam is implemented only in its high Reynolds number version. ILES simulations are run with meshes of 18000,31000 and 50000 cells. For the parameters of interest in this work, mesh independency was reach with meshes larger than 31000 cells, so that most results presented hereafter correspond to this mesh resolution. In order to avoid a strong refinement of the mesh at the wall, a wall function is also applied through an adjustment of viscosity in the raw of cells adjacent to the wall, as in [1].

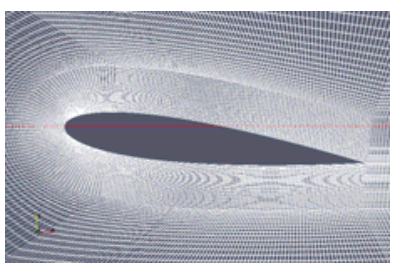

$\mathrm{k}-\omega \mathrm{SST}$

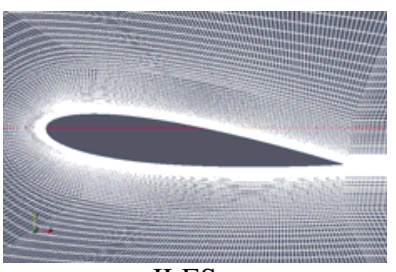

ILES
Figure 1: Baseline meshes for RANS and ILES simulations

The simulations are first run in single phase until convergence. The cavitation model is activated afterwards, reducing slowly the vapor pressure until reaching the desired value of $\sigma$. The time step is automatically adjusted, with a maximum CFL number ranging from 0.5 to 0.1 according to the value of $\sigma$.

\section{RESULTS AND DISCUSSION}

In the simulations presented hereafter, the cavitation number $\sigma$ is varied between 0.5 and 2, while the angle of attack $\alpha$ ranges between 2 and $10^{\circ}$. The different conditions simulated in this work are depicted as dots on the cavitation mapping established by Kjeldsen et al. [11] in figure 2 .

Two cases of sheet cavitation are analyzed in details here: case 1 at the limit of patch cavitation $\left(\sigma=1.5, \alpha=7^{\circ}\right)$ and case 2 in the 


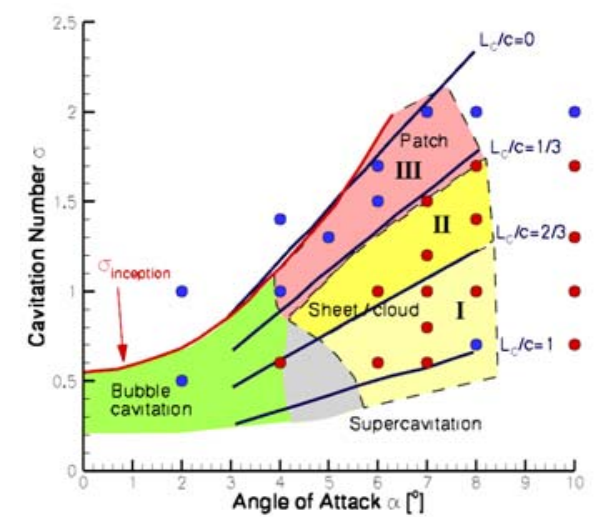

Figure 2: Cavitation mapping on a NACA0015 foil with the different types of oscillations according to Kjeldsen et al. [11]. $L_{c} / c$ is the relative cavity length. The dots represent the conditions simulated in this paper.

very dynamic regime of sheet/cloud $\left(\sigma=1, \alpha=7^{\circ}\right)$. In case 1 , it was no surprise observing a complete stabilization of the cavity with the standard k- $\omega$ SST model. The cavity remains very thin and attached at the leading edge of the foil. It can be observed that in the rear part of the cavity, where vapor is mixed with liquid, turbulent viscosity is especially high, which impedes the reentrant jet from being effective. A modification is thus made in the formulation of the turbulent viscosity, following the idea of Coutier-Delgosha et al. [6]:

$$
\mu_{t}=\frac{f(\rho) k}{\omega} \frac{1}{\max \left(\frac{1}{\alpha *}, \frac{S F 2}{a_{1} \omega}\right)} \text { with } f(\rho)=\rho_{v}+\alpha^{n}\left(\rho_{l}-\rho_{v}\right)
$$

where the function $\mathrm{f}(\rho)$ replaces $\rho$ in the original formulation. The exponent $n$ is taken equal to 10 , according to the recommendations in [6]. In that way, the density tends to the one of pure vapor and pure liquid when $\gamma=0$ and 1 respectively, while it keeps a low value in the intermediate range of $\gamma$. In spite of this change, the cavity remains stable in case 1 , as evidenced in figure 3 (left hand side). In contrast, ILES succeeds in capturing a periodic shedding of the cavity (figure 3 , right hand side). The power spectra of the lift coefficient $C_{l}$ and of the cavity length $L_{c}$ show indeed a much more energetic peak with ILES (figure 4).

With all turbulence models, the cavity length reaches about $1 / 3$ of the chord length, in agreement with the mapping in figure 2 . It should be noted that case 1 is at the limit between patch and sheet cavitation, which corresponds to type III oscillations according to Kjeldsen et al. This type of oscillations are believed to be dominated by the reentrant jet mechanism, which would mean that the turbulent viscosity reduction is not quite sufficient for the reentrant jet to be effective once the first oscillating cycle has passed (during which cavity shedding is observed). A strong mesh refinement at the wall did not improve the predictions of the reentrant jet. Nevertheless, the spectral peak corresponding to type III oscillations is known to be weaker than in type II oscillations which correspond to the more dynamic sheet/cloud cavitation. For that reason, it is believed that the dynamics is too weak in the patch cavitation regime to be captured by RANS models.

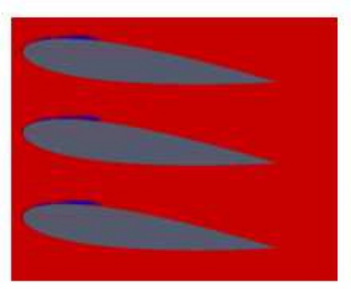

Modified k- $\omega$ SST

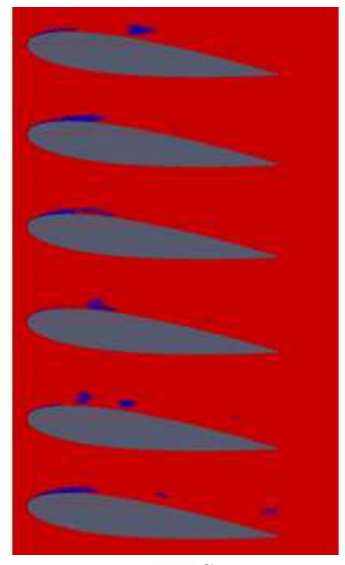

ILES

Figure 3: Comparison of vapour fraction fields with ILES and k$\omega \operatorname{SST}\left(\sigma=1.5, \alpha=7^{\circ}\right)$. Images are separated by $\Delta t=0.004 \mathrm{sec}$.

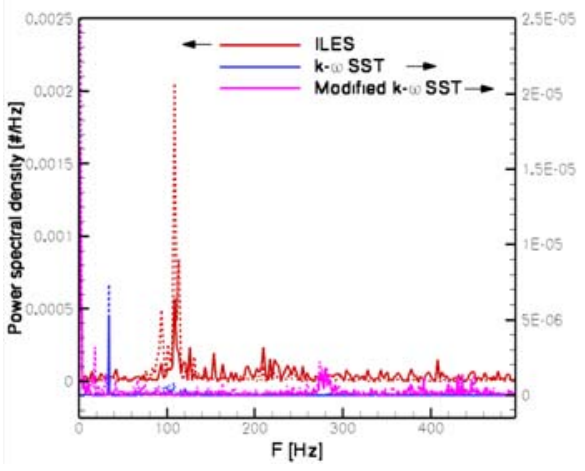

Figure 4: Power spectra of the lift coefficient $C_{l}$ (solid lines) and attached cavity length $L_{c}$ (dotted lines) with the different turbulence models in case $1\left(\sigma=1, \alpha=7^{\circ}\right)$.

In case 2 , the cavity behavior obtained with ILES and the modified $\mathrm{k}-\omega \mathrm{SST}$ is in better agreement (figure 5). It should be noted that in that regime of pure sheet/cloud cavitation, the standard k- $\omega$ SST still does not succeed in predicting the unsteadiness of the cavity: after a few cycles of cloud shedding, the cavity stabilizes. As it can be observed in figure 5, the cavity shedding cycles and the resulting formation of vapor clouds is similar with both models. The edge of the clouds is sharper and smoother with the RANS model, due to the fact that smaller scale phenomena are not resolved; however, it does not affect too much the periodic mechanism.

A total of 26 simulations are conducted for a wide variety of conditions with ILES, as depicted in figure 2: the dots added on the cavitation map of Kjeldsen et al. correspond to the different simulations performed. Red dots denote the conditions in which a clear periodic behavior of cavitation was detected with ILES, 


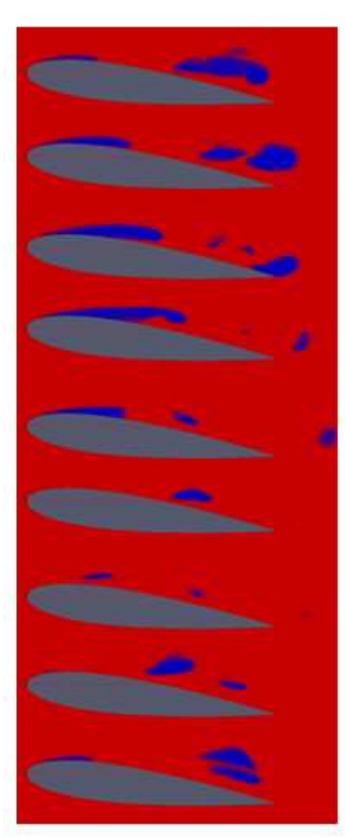

Modified k- $\omega$ SST

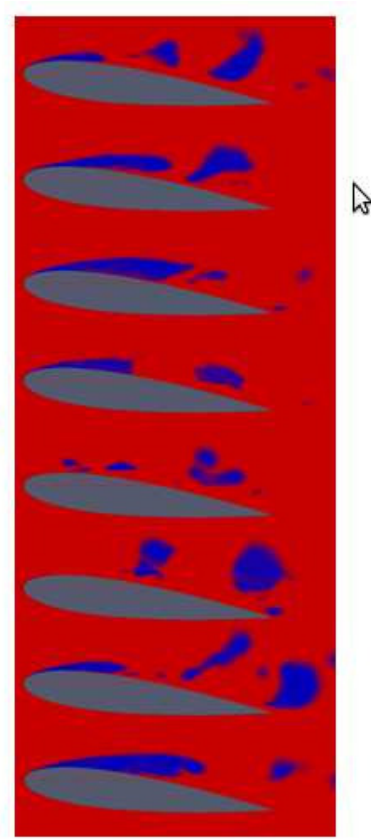

ILES
Figure 5: Comparison of a cavity shedding cycle with ILES and $\mathrm{k}-\omega \operatorname{SST}\left(\sigma=1, \alpha=7^{\circ}\right)$. Images are separated by $\Delta t=0.004 \mathrm{sec}$.

while blue dots stand for conditions in which no periodicity is observed. All periodic behaviors are found to be in the sheet/cloud regime, corresponding to types I and II oscillations as defined by Kjeldsen. It can be noticed that even with ILES, type III oscillations in the patch cavitation regime are hardly captured. A dominant low frequency is sometimes found, in agreement with the experimental observations of Kjeldsen et al., but the computation time is not long enough to conclude about it. In addition, the two-dimensionality of the simulations probably hides the mechanism. Close to supercavitation, one case $\left(\sigma=0.7, \alpha=8^{\circ}\right)$ shows no particular periodic behavior. This has already been observed by Coutier-Delgosha et al. [7]: when the cavity terminates right at the trailing edge of the foil, instability disappears.

Although the period of cavity shedding seems to be similar on the sequences of figure 5, the characteristic frequencies $F$ found in the $C_{l}$ spectra are systematically lower with the modified k- $\omega$ SST than with ILES. This can be seen in figure 6 , which gathers the characteristic Strouhal numbers $S t=F c / U$ based on the chord length as a function of the parameter $\sigma 2 \alpha$. In case 2 for example $(\sigma / 2 \alpha=4)$, the Strouhal number is only a bit lower with the modified k- $\omega$ SST than with ILES, but the difference is larger at the other values of $\sigma / 2 \alpha$ simulated. Besides, it can be noted that the Strouhal numbers obtained with ILES are in very good agreement with experimental data [12]; one can observe perfectly the transition between type I oscillations for $\sigma 2 \alpha \leq 4$, characterized by a fairly constant value between 0.15 and 0.3 , and type II oscillations, characterized by higher although less energetic frequencies, which vary linearly with $\sigma$. As already said, type III oscillations have not been clearly identified.

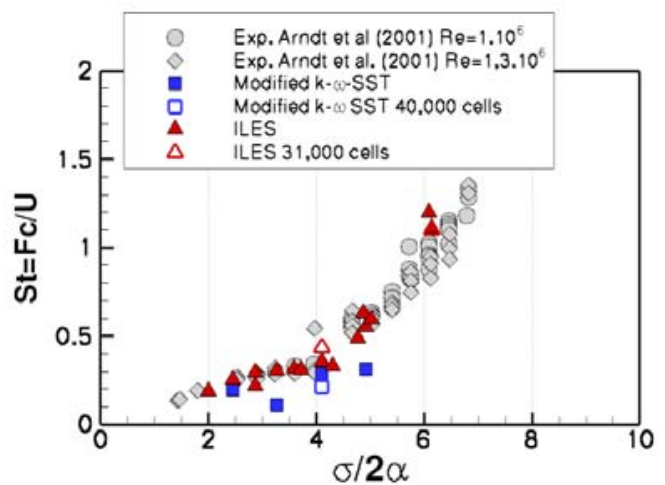

Figure 6: Dimensionless dominant frequency versus $\sigma 2 \alpha$.

It seems thus that the frequencies predicted by the modified $\mathrm{k}-\omega$ SST model are slightly underestimated. A reason for that is visible in figure 5: the cavity volume is larger in general with ILES than with $\mathrm{k}-\omega$, which provokes an earlier shedding of vapour clouds. This is confirmed by the time evolution of the attached and shed cavity volume in figure 7 . The attached cavity grows faster with ILES, which leads to a faster cloud detachment and thus a larger volume of the shed cavities.
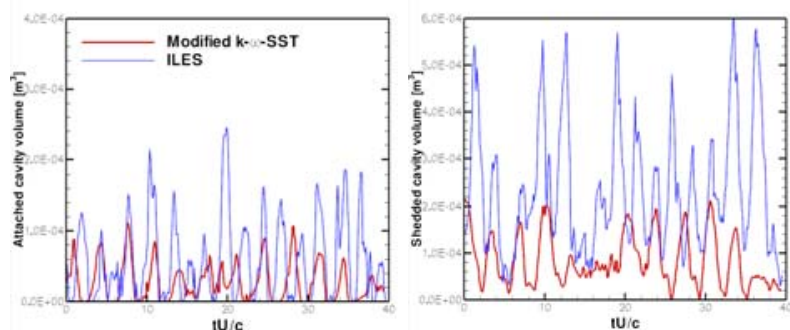

Figure 7: Time evolution of the attached (left) and shed (right) cavity volumes in case $2\left(\sigma=1, \alpha=7^{\circ}\right)$.

The mean cavity volumes are indicated in table 1 for cases 1 and 2. It should be noted that the characteristic Strouhal numbers of the attached and shed cavity volume signals are almost equal and in agreement with the dimensionless frequencies found in the spectra of $C_{l}$ in figure 6.

\begin{tabular}{|c|c|c|c|c|c|}
\hline & $\begin{array}{l}\text { Turbulen } \\
\text { ce model }\end{array}$ & $\begin{array}{l}\text { Total cavity } \\
\text { vol. } \quad\left[10^{-4}\right. \\
\left.\mathrm{m}^{3}\right]\end{array}$ & $\begin{array}{l}\text { Attached } \\
\text { cavity vol. } \\
{\left[10^{-4} \mathrm{~m}^{3}\right]}\end{array}$ & $\begin{array}{l}\text { Shed cavity } \\
\text { vol. } \quad\left[10^{-4}\right. \\
\left.\mathrm{m}^{3}\right]\end{array}$ & St \\
\hline \multirow{2}{*}{$\begin{array}{l}\sigma=1.5 \\
\text { (case 1) }\end{array}$} & k- $\omega$ SST & 0.21 & 0.20 & 0.01 & I \\
\hline & ILES & 0.30 & 0.09 & 0.21 & 1.10 \\
\hline \multirow{2}{*}{$\begin{array}{l}\sigma=1 \\
\text { (case 2) }\end{array}$} & k- $\omega$ SST & 1.1 & 0.3 & 0.8 & 0.29 \\
\hline & ILES & 2.9 & 0.6 & 2.3 & 0.35 \\
\hline
\end{tabular}

Table 1: Mean cavity volumes with ILES and k- $\omega$ SST models.

This discrepancy in the cavity volumes observed with different turbulence models while keeping the mass transfer coefficients constant, is symptomatic of a tight coupling between the phase change and turbulent mechanisms. There are probably some 
small scale turbulent fluctuations that the RANS model cannot capture, and which promote mass transfer.

The next step in investigating the consequences of underestimating (or overestimating) the cavity volume is to check its effect on the mean lift and drag coefficients, as well as their RMS values. For $\alpha=7^{\circ}$, the evolution of the mean coefficients $C_{l}$ and $C_{d}$ is presented in figure 8, together with the experimental data of Amromim et al. [13]. It can be noted that ILES predicts qualitatively well the evolution of $C_{l}$, marked by a plateau between $\sigma=1.5$ and 1 (type II oscillations) and an abrupt decrease below $\sigma=1$ (type I). The plateau region corresponds to regimes where the cavity is relatively short $\left(L_{c} / c<1 / 3\right)$, but as it detaches periodically, it does not affect too much the mean value of $C_{l}$. At lower $\sigma$, a longer cavity survives at the leading edge, and although it is regularly shed, it ends up affecting the mean $C_{l}$. With the k- $\omega$ SST model, a continuous decrease of $C_{l}$ is observed from $\sigma=2$; this is because in the region $2 \leq \sigma \leq 1$ where type II oscillations should occur, the cavity remains attached permanently, which makes the mean value of $C_{l}$ decrease. When one enters in type I oscillations ( $\sigma$ $\leq 1), C_{l}$ gets closer to the value predicted by ILES, as the cavity gets longer and the shedding cycle begins. The systematic underestimation of $C_{l}$ with respect to experiments is probably due to the $2 \mathrm{D}$ character of simulations. It has already been observed by Kunz et al. [9]. The evolution of the mean drag coefficient $C_{d}$ is reasonably well captured with both turbulence models, although the RANS model dos not predict the slight overshoot at $\sigma=1$.
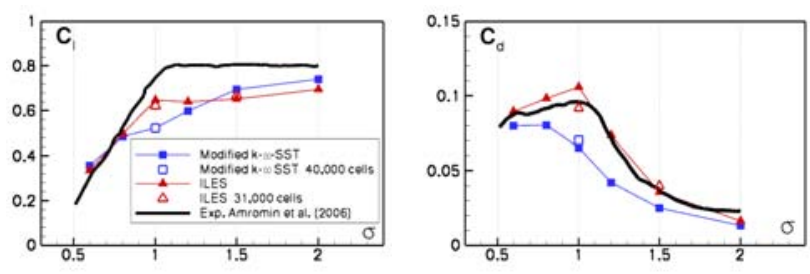

Figure 8: Mean lift and drag coefficients for $\alpha=7^{\circ}$.
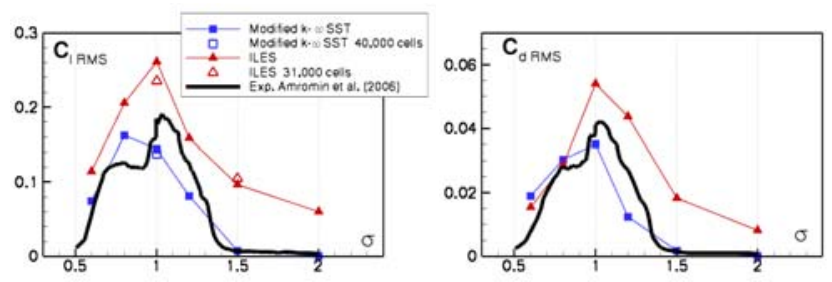

Figure 9: RMS lift and drag coefficients for $\alpha=7^{\circ}$.

The evolution of the RMS of $C_{l}$ and $C_{d}$ with $\sigma$ is presented in figure 9. The qualitative shape of the curves is well predicted by both models: they reach a maximum in the sheet/cloud regime $(\sigma=1)$ before decreasing abruptly as one approaches supercavitation. ILES seems to overestimate a bit the amplitude of fluctuations, it is probably due to a lack of turbulent dissipation. That would explain that the cavities are able to grow during a longer time with ILES. It is all the more obvious for the larger values of $\sigma(\sigma \geq 1.5)$, for which the RMS values of both drag and lift are overestimated of almost one order of magnitude. Although in this cavitation regime, ILES allows capturing the correct frequencies of type II oscillations detected experimentally (figure $6, \sigma / 2 \alpha \geq 4$ ), it overestimates their amplitude. The RMS values yielded by the RANS model for $\sigma \geq 1.5$ are in much better agreement with experiments. Of course, these problems could be fixed using a genuine LES model as in [2], but it involves CPU requirements that are beyond our grasp now.

The under or overestimation of cavity volumes can have important consequences on the downstream evolution and collapse of vapour clouds. This point is of primary importance, as it will determine the risk of erosion. As it appears in figure 5, the shed cavity volumes are substantially higher with ILES than with RANS. In order to evaluate the rate of collapse of vapour clouds along the foil, three vertical lines are placed at distances of respectively $0.6,0.8$ and $1 c$ from the trailing edge (see figure 10 ), and the vapour volume passing through the lines is monitored as a function of time. This allows determining the frequency of passage of clouds at different locations along the foil, and evaluating their mean volume, which is an indication of how strong the collapse might be.

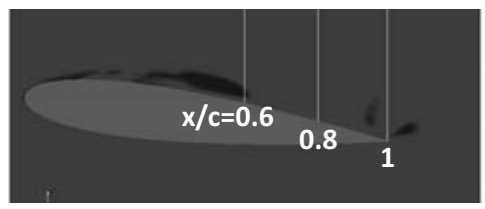

Figure 10: Position of the 3 line counters along the foil.

It can be seen in figure 11 that as expected, the number of cavities decreases as one approaches the trailing edge. The difference between the frequency of cavities crossing one line and the frequency of cavities crossing a downstream position thus gives an idea of the collapse frequency of vapour clouds between these two points. In case 2 for example, counting the number of clouds passing across lines 1 and 3 , one finds a collapse frequency of about $17 \mathrm{~Hz}$ (corresponding to $S t=0.18$ ) between the two lines. Nevertheless, it is difficult to evaluate the number of cavities passing through line 1; vapour clouds have just been shed in this zone, and they tend to merge easily together. The passage of smaller cavities is easier to detect at the trailing edge (figure 11). Even if cloud collapse is obvious from the time signals, the corresponding spectra all display a dominant frequency which corresponds to the shedding frequency. So even though collapse takes place along the foil, cloud travelling is still controlled by the mechanism of cavity shedding.

As summarized in table 2, the mean volume of travelling cavities is larger with ILES. At $\sigma=1$, it tends to increase as one approaches the trailing edge, due to cavity merging. At the lower $\sigma=1.2$, clouds tend to remain independent and the mean volume is rather decreasing as a result of progressive collapse. 

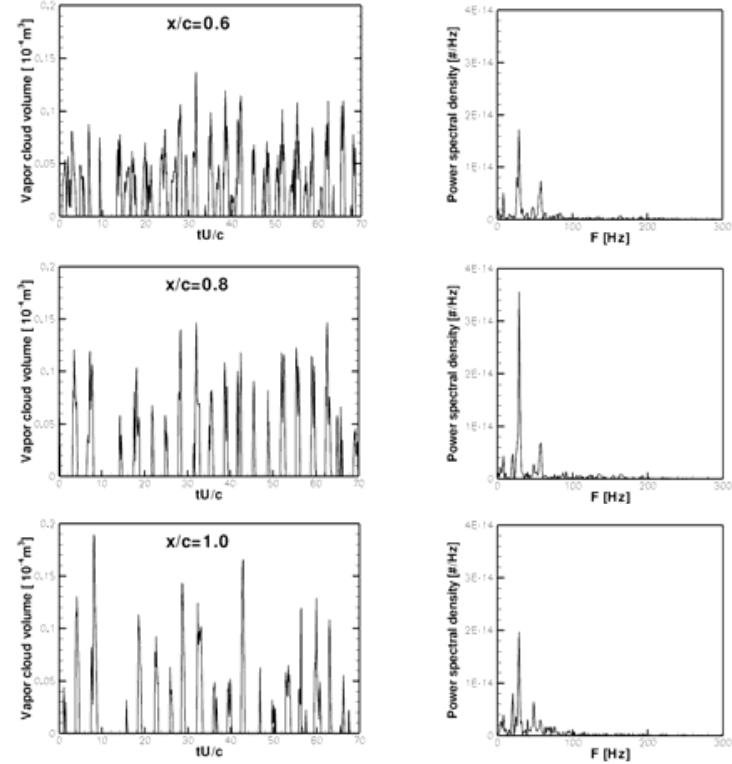

Figure 11: Time signals of the volume of vapour clouds passing through the 3 lines (left) and corresponding spectra (right) obtained with the k- $\omega$ model, case $2\left(\sigma=1, \alpha=7^{\circ}\right)$.

\begin{tabular}{|l|c|c|c|c|}
\hline & $\begin{array}{c}\text { Turbulence } \\
\text { model }\end{array}$ & $\begin{array}{l}\text { Mean cavity } \\
\text { vol. } \begin{array}{c}\text { through } \\
\text { line } 1\left[10^{-4} \mathrm{~m}^{3}\right]\end{array}\end{array}$ & $\begin{array}{l}\text { Mean cavity } \\
\text { vol. through } \\
\text { line 2 }\end{array}$ & $\begin{array}{l}\text { Mean cavity } \\
\text { vol. through } \\
\text { line 3 }\end{array}$ \\
\hline \multirow{2}{*}{$\sigma=1.2$} & $\mathrm{k}-\omega$ SST & 0.19 & 0 & 0 \\
\cline { 2 - 5 } & ILES & 0.38 & 0.36 & 0.16 \\
\hline \multirow{2}{*}{$\begin{array}{l}\sigma=1 \\
\text { (case 2) }\end{array}$} & $\mathrm{k} \omega \mathrm{\omega SST}$ & 0.30 & 0.40 & 0.31 \\
\cline { 2 - 5 } & ILES & 0.43 & 0.47 & 0.57 \\
\hline
\end{tabular}

Table 2: Mean cavity volumes with ILES and k- $\omega$ SST models.

Some of the travelling clouds end up on the surface of the foil; those are particularly dangerous in terms of erosion. The volume of these clouds was also monitored in time, but the corresponding spectra did not display any dominant frequency. Their mean volume is equivalent to 1 to $10 \%$ of the total cloud volume, depending on the conditions.

It is important to note that although our comparative study brings some interesting information about the difference between ILES and RANS in 2D, some additional discrepancies are to appear in 3D: de Lange et al. [14] notes for example that in sheet cavitation, smaller cavities are shed in 2D than in 3D. Evidently the strong vorticity generated during shedding will not affect the travelling clouds in the same way in $2 \mathrm{D}$ than in 3D.

\section{SUMMARY}

The periodic phenomena on a partially cavitating 2D NACA0015 foil are studied in a systematic manner in this paper. The formation and travelling of vapor clouds is proved to be directly related to the mechanism of cavity shedding in the sheet cavitation regime. It is shown that the use of ILES for turbulence modeling on a relatively coarse grid allows capturing the main periodic phenomena, while a k- $\omega$ SST predicts the occurrence of cavity shedding in the fully sheet/cloud regime only if turbulent viscosity is reduced in the vapor-liquid mixture. The volume of cavities is a bit underestimated with the RANS model, which leads to an underestimation of the characteristic frequency. However, the CPU time being one order of magnitude larger with ILES than with RANS, it would be interesting to investigate the weaknesses of the k- $\omega$ SST in 3D in order to be able to use it in fully $3 \mathrm{D}$ configurations.

\section{ACKNOWLEDGMENTS}

The financial support of the Galician Region (grant \#09DPI025CP) and the technical support of CESGA are gratefully acknowledged.

\section{REFERENCES}

[1] Bensow, R. \& Bark, G. 2010, "Simulating cavitating flows with LES in OpenFoam," V European Conf. on Computational Fluid Dynamics ECCOMAS CFD 2010.

[2] Kim, S. E. 2009, "A numerical study of unsteady cavitation on a hydrofoil," Proc. CAV2009, Paper 56.

[3] Kuiper, G. 2010, "New developments and propeller design," $9^{\text {th }}$ Int. Conf. on Hydrodynamics.

[4] Goncalvès, E. 2010, "Numerical study of unsteady cavitating flows" Eur. J. Mechanics B/Fluids 30:26-40.

[5] Bernsten, G. S., Kjeldsen, M. \& Arndt, R. E. A. 2001, "Numerical modeling of sheet and tip vortex cavitation with Fluent 5," Proc. CAV 2001, Paper B5.006.

[6] Coutier Delgosha, O., Fortes-Patella, R. \& Reboud, J.L. 2003, "Evaluation of the turbulence model influence on the numerical simulations of unsteady cavitation," $J$. Fl. Eng. 125:38-45.

[7] Coutier Delgosha, O., Deniset, F., Astolfi, J.A. \& Leroux, J.-B. 2007, "Numerical prediction of cavitating flow on a two-dimensional symmetrical hydrofoil and comparison to experiments," J. Fl. Eng. 129:279-292.

[8] Pouffary, B., Fortes-Patella, R. \& Reboud, J.L. 2003, "Numerical simulation of cavitating flow around a 2D hydrofoil : a barotropic approach," Proc. CAV03.

[9] Kunz, R., Lindau, J., Kaday, T. \& Peltier, L. 2003 "Unsteady RANS and detached eddy simulation of cavitating flow over a hydrofoil", Proc. CAV03.

[10] Schnerr, G. H. \& Sauer, J. 2001 "Physical and numerical modeling of unsteady cavitation dynamics," Proc. $4^{\text {th }}$ Int. Symp. Multiphase Flows, New Orleans (USA).

[11] Kjeldsen, M., Arndt, R. \& Effertz, M. 2000, "Spectral characteristics of sheet/cloud cavitation," J. Fl. Eng. 122:481-487.

[12] Arndt, R., Song, C., Kjeldsen, M., \& Keller, A. 2001 Instability of partial cavitation: Numerical/ experimental approach," Proc. $23^{\text {rd }}$ Symp. on Naval Hydrodynamics.

[13] Amromim, E., Kopriva, J., Arndt, R. E. A., Wosnik, M. 2006, "Hydrofoil drag reduction by partial cavitation," J. Fl. Eng. 128:931-936.

[14] De Lange, D. F. \& de Bruin, G. J. 1998, "Sheet cavitation and cloud cavitation, re-entrant jet and three dimensionality," Appl. Sci. Res. 58: 91-114. 